\title{
Relación entre la producción de biogás y biol a partir de restos de trucha y estiércol vacuno
}

The relationship between the production of biogas and biol from trout waste and cow manure

Olenka Garavito ${ }^{* \bullet}$ y Luis Gomero ${ }^{\circledR}$

1 Facultad de Ciencias Ambientales, Universidad Científica del Sur. Lima, Perú.

2 Red de Acción en Agricultura Alternativa (RAAA). Lima, Perú.

\section{SOUTH} SUSTAINABILITY

Citar como: Garavito, O. y Gomero, L. (2020). «Relación entre la producción de biogás y biol a partir de restos de trucha y estiércol vacuno». South Sustainability, 1(1), e008 DOI: 10.21142/SS-0101-2020-008

Artículo recibido: 23/12/2019 Arbitrado por pares

Artículo aceptado: 29/5/2020

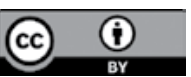

(c) Los autores, 2020. Publicado por la Universidad Científica del Sur (Lima, Perú)

${ }^{*}$ E-mail de correspondencia: olenkagr94@gmail.com

\section{RESUMEN}

En el presente estudio se ha analizado la relación que existe entre la producción de biogás y biol a partir de las mezclas de restos de trucha y estiércol vacuno. Los tratamientos evaluados presentaron diferentes cantidades de estiércol vacuno T1 (6 kg), T2 (12 kg) y T3 (24 kg). La mayor producción de biogás y composición del biol se dio en el siguiente orden descendente T3 > T2 > T1 > Control, y fue el tratamiento T3 el que obtuvo resultados más elevados y óptimos para ambas variables. Con respecto a la cantidad de biogás acumulado para este tratamiento, se produjo un total de 159,3 L; asimismo, la composición del biol estuvo conformada por la concentración de nitrógeno, fósforo, potasio, calcio, magnesio y sodio, que fue $6600,565,89,1136,67,1603,33,563,33$ y $1408,33 \mathrm{mg}^{l^{-1}}$, respectivamente; además de la cantidad de ácidos húmicos, ácidos fúlvicos y huminas 1,06 \%; 1,39\%; $0,48 \%$, respectivamente.

Palabras clave: biol, biogás, estiércol, restos de trucha

\section{ABSTRACT}

This study analyzed the relationship between the production of biogas and biol from trout waste and cow manure. The treatments evaluated contained different amounts of manure, T1 (6 kg), T2 (12 kg) and T3 $(24 \mathrm{~kg})$, of which the treatments which produced the highest quantity of biogas and biol were T3>T2>T1>Control, in descending order. Therefore, it was shown that the T3 treatment produced the best results for both variables. With regard to the amount of accumulated biogas for this treatment, total production was $159.3 \mathrm{l}$. The composition was defined by concentrations of nitrogen, potassium, calcium, magnesium and sodium $(6600,565.89,1136.67,1603.33,563.33$ and $1408.33 \mathrm{mg} . \mathrm{l}^{-1}$, respectively), while the percentages of humic and fulvic acids, and humins were $1.06 \%$, $1.39 \%, 0.48 \%$, respectively.

Keywords: biol, biogas, manure, trout waste 


\section{Introducción}

Las empresas acuícolas productoras de truchas han incrementado su producción en el transcurso de los años a nivel Perú en 10500 t (Plan Nacional de Desarrollo Acuícola, PNDA, 2010) y con ello ha aumentado la generación de residuos orgánicos, lo que representa el $11,4 \%$. Su disposición final son los botaderos, al aire libre y en algunos casos en un relleno sanitario (Ministerio del Ambiente, Minam, 2013).

A su vez, la producción orgánica en el sector agroexportador se ha incrementado a nivel Perú en un $25 \%$ con respecto a 2014, y se maneja bajo los principios de agricultura orgánica un aproximado de 607872 hectáreas por año, que ahora requieren insumos alternativos a los fertilizantes sintéticos (Servicio Nacional de Sanidad Agraria, Senasa, 2015).

Debido a su alto contenido en materia orgánica, los residuos acuícolas y las excretas de vacunos son materiales potencialmente aprovechables como alternativa para la obtención de energía y la producción de abonos. Uno de estos subproductos es el biogás, que representa el 0,07 \% de la producción de energía eléctrica a nivel Perú (Comité de Operaciones del Sistema Interconectado Nacional, COES, 2015).

Otro de los subproductos de este proceso es la producción de biol, usado para aumentar el crecimiento vegetativo de los cultivos, que actualmente es producido en diferentes proyectos y empresas exitosas a nivel Perú, como Bioagricultura Casa Blanca, Care Perú y Fundo Los Tayas, que producen más de 2000 litros diarios de biol (Ministerio de Agricultura y Riego, Minagri, 2011).

La presente investigación analizó la relación que existe entre la producción de biogás y biol a partir de las mezclas de restos de la crianza de trucha y el estiércol de ganado vacuno.

Este análisis se basó en la cantidad de litros de biogás producidos por cada tratamiento y en la concentración de macronutrientes del biol, como nitrógeno, fósforo, potasio, calcio, magnesio y sodio, así como la concentración de ácidos húmicos, ácidos fúlvicos y huminas que presentó cada uno de los tratamientos analizados, que consistieron en diferentes cantidades de restos de trucha mezclados con estiércol vacuno que usaron un modelo de biodigestor tipo batch de régimen discontinuo.

Existen diversos tipos de biodigestores, que se definen principalmente en función de las características del material o sustrato fermentable y régimen de carga de este sustrato. Uno de los biodigestores que tiene bastante potencial para las investigaciones son los sistemas batch, debido a que se cargan una sola vez, es decir, son de régimen discontinuo. Durante la operación de este tipo de biodigestor se busca mantener el $\mathrm{pH}$ en condiciones casi neutrales, para que ocurra la fermentación en un periodo de 30 a 180 días, dependiendo de la temperatura ambiental donde se instalen estos reactores. En este periodo la producción diaria de gas tenderá a aumentar hasta un máximo y luego comenzará a decaer (FAO, 2011).

\section{Materiales y métodos}

\section{Localización, duración, materiales usados}

El estudio se realizó en el distrito de Carabayllo, de Lima Metropolitana, de febrero a abril del 2017, durante 70 días. La materia prima usada para la elaboración del biol y la producción de biogás fueron los restos de trucha provenientes de la empresa Peruvian Andean Trout S. A. C. (Patsac), compuestos por cabeza, aletas, piel y vísceras, que representaban alrededor de un $47 \%$ del peso de los peces (Contreras, 1994) y cuerpos de truchas que han muerto durante la crianza (Centro de Estudios para el Desarrollo y la Participación, Cedep, 2009).

El otro sustrato utilizado para la producción de biol y biogás fue el estiércol vacuno, que contiene nutrientes que pueden ser usados como fertilizantes, pero se necesita un tratamiento previo para eliminar patógenos, larvas o huevos dañinos para la salud. Asimismo, permite que los nutrientes sean más accesibles para las plantas. Este tratamiento se realiza mediante la digestión anaeróbica. Los microorganismos que degradan materia orgánica en condiciones de ausencia de oxígeno producen residuos útiles y sin riesgo de transmisión de enfermedades (Soria et al., 2001).

\section{Diseño del biodigestor, volumen y carga, ensayos analizados, parámetro de control y producción de biogás}

Previo al inicio de la etapa experimental, se construyeron 12 biodigestores instalados bajo el diseño tipo batch, como se muestra en la figura 1.

Los biodigestores construidos se distribuyeron en tres tratamientos y un control, y cada uno contó con tres repeticiones. La capacidad de los biodigestores fue de 90 litros. Considerando que se debe tener un borde libre de 15 a $20 \mathrm{~cm}$ para facilitar la digestión anaerobia (Restrepo, 2001), se procedió a llenar los tratamientos hasta los 75 litros. Previamente el estiércol vacuno tuvo una dilución, como se indica en la distribución mostrada de la tabla 1.

Para garantizar que la digestión anaerobia contara con las condiciones óptimas se realizó un monitoreo de $\mathrm{pH}$ y temperatura. Se evaluaron ambos parámetros al sacar una muestra del biol en vasos descartables, y se hizo una lectura de estos análisis mediante el uso de un equipo con una frecuencia de dos veces por semana durante las 10 semanas que duró la fase experimental (Castillo, 2012).

Asimismo, se realizaron análisis iniciales de la mezclas y finales para determinar la composición del biol, tal como se muestran en la figura 2.

Estos análisis fueron realizados por el laboratorio de análisis de suelos, aguas y fertilizantes de la misma universidad, para la determinación de macronutrientes y ácidos húmicos y fúlvicos. 


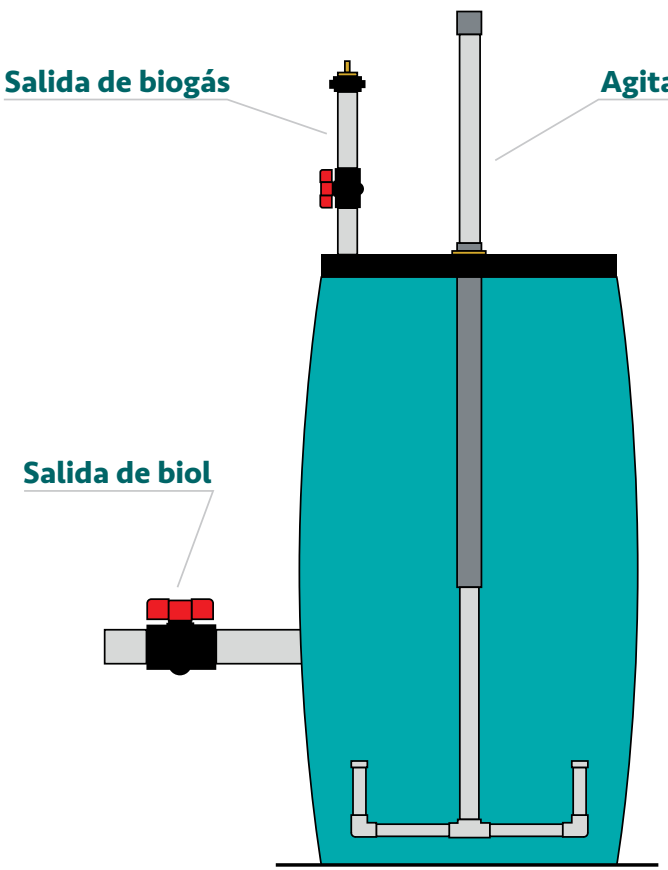

Figura 1. Diseño del biodigestor utilizado en el ensayo. Fuente: Castillo (2012)

Finalmente, a fin de calcular la producción de biogás durante las 10 semanas que duró la fase experimental, se utilizaron flotadores para el almacenamiento del biogás por cada tratamiento, tomando en cuenta que cada vez que se encontraban repletos se desplazó el biogás acumulado a un tanque lleno de agua siguiendo el principio de Arquímedes, para que el volumen de agua se desplazara de este recipiente a otro graduado y directamente relacionado con el volumen del biogás producido (Castillo, 2012).

El tanque de medición de biogás producido tiene dos mangueras instaladas, una para el ingreso del biogás proveniente del flotadory la otra para la disposición del agua desplazada. Esta última manguera se colocó sumergida hasta la base del tanque. De esta manera la presión del gas desplazó el agua a otro envase graduado (Palomino, 2007).

\section{Análisis estadístico}

El trabajo realizado fue experimental y se realizó bajo un diseño completamente al azar (DCA) con cuatro tratamientos y tres repeticiones de cada uno.

Tabla 1. Distribución de tratamientos

\begin{tabular}{cccc}
\hline & & \multicolumn{2}{c}{ Estiércol vacuno diluido } \\
\cline { 3 - 4 } Tratamientos & $\begin{array}{c}\text { Restos de } \\
\text { trucha (L) }\end{array}$ & $\begin{array}{c}\text { Estiércol } \\
\text { vacuno (kg) }\end{array}$ & Agua (L) \\
\hline C & 75 & - & - \\
\hline T1 & 66 & 6 & 3 \\
\hline T2 & 57 & 12 & 6 \\
\hline T3 & 39 & 24 & 12
\end{tabular}

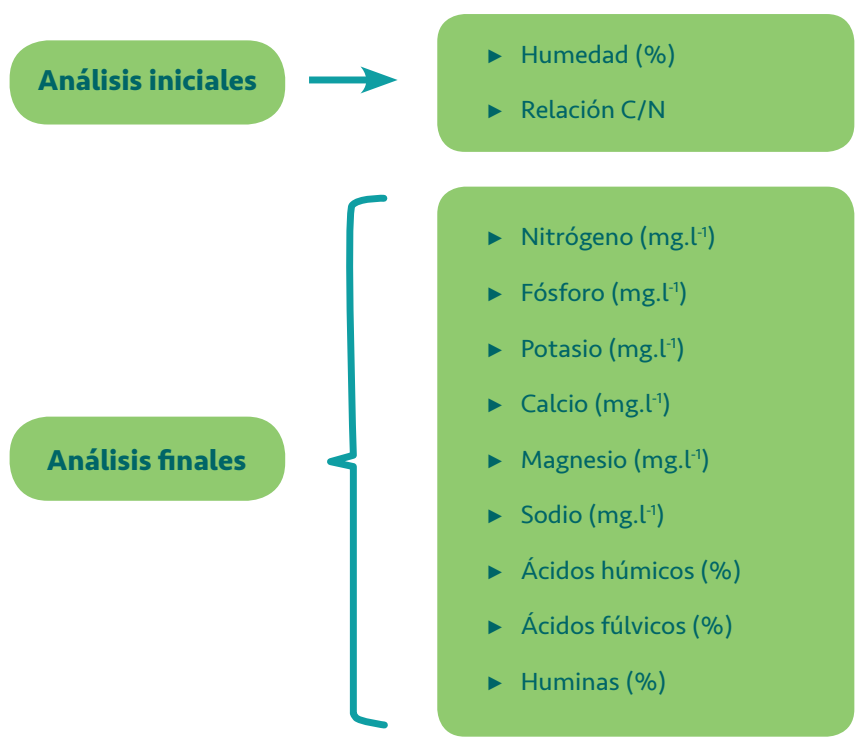

Figura 2. Análisis realizados a la composición del biol

Se usó el programa SPSS 21 y se realizaron análisis de varianza (Anova) para cada variable obtenida de los bioles (nitrógeno, fósforo, potasio, calcio, magnesio, sodio, ácidos húmicos, ácidos fúlvicos y huminas). Del mismo modo, se realizó un análisis de varianza para los valores de $\mathrm{pH}$ y temperatura, que se midieron dos veces a la semana durante la fase experimental del proyecto. Además, considerando que sí se tuvieron valores significativos, se procedió a realizar pruebas de Tukey para determinar los valores altos, medios y bajos para cada variable.

A fin de determinar la relación entre la producción de biogás y las concentraciones de nutrientes de los bioles por cada tratamiento, se realizó el análisis de correlación de Pearson con un intervalo de confianza del $95 \%$. Se consideraron los valores de $p<0,05$ como significativos. Por consiguiente, existe una relación entre biogás y concentración de nutrientes.

\section{Resultados: caracterización de los resultados, comportamiento del $\mathrm{pH}$ y temperatura}

Los valores de la relación carbono-nitrógeno son inferiores a 30, valor considerado como óptimo para el proceso de fermentación anaeróbica (tabla 2).

Tabla 2. Caracterización inicial de tratamientos

\begin{tabular}{ccc}
\hline Tratamientos & Humedad (\%) & $\mathbf{C / N}$ \\
\hline C & 95,81 & 6,2 \\
\hline T1 & 93,45 & 7 \\
\hline T2 & 92,56 & 11,1 \\
\hline T3 & 85,11 & 21,7 \\
\hline
\end{tabular}


A su vez, el comportamiento del pH por cada tratamiento durante las 10 semanas de duración de la investigación presenta diferencias significativas (tabla 3), en función del Anova de un factor: $F(3,236)=50,449$, $\mathrm{p}<0,01$. La prueba de Tukey demuestra que el control resultó significativamente distinto en comparación con el tratamiento 1 , el tratamiento 2 y el tratamiento 3. De la misma forma sucedió para el tratamiento 1, el tratamiento 2 y el tratamiento 3, al hacer la comparación entre tratamientos (ver tabla 3).

Por otro lado, para el comportamiento semanal de la temperatura $\left(26 \pm 4{ }^{\circ} \mathrm{C}\right)$ no se encontró diferencia significativa entre los tratamientos con el comportamiento semanal de la temperatura $(F(3,236)=0,470, p=0,704)$.

\section{Producción de biogás}

Se tiene que la producción de biogás total por cada tratamiento en orden decreciente es T3 $>$ T2 > T1 > Control, con valores de 159,30; 121,57; 95,57 y 72,63 litros. El análisis estadístico a los resultados totales de la producción de biogás obtuvo diferencias estadísticas significativas entre tratamientos, como se observa en la tabla 4.

\section{Composición del biol obtenido}

Asimismo, se determinó la cantidad de macronutrientes por cada uno de los tratamientos y sus repeticiones. Se obtuvieron promedios y su significancia, como se observa en la tabla 5.

Tabla 3. Resultados del pH de los tratamientos recogidos a lo largo de 10 semanas

\begin{tabular}{cccc}
\hline \multirow{2}{*}{ Tratamientos $^{\mathrm{a}}$} & \multicolumn{3}{c}{ Resultados de $\mathrm{pH}$ por tratamiento } \\
\cline { 2 - 4 } & Media & SE & Tukey \\
\hline $\mathrm{C}$ & 6,95 & 0,03 & $\mathrm{a}$ \\
\hline $\mathrm{T} 1$ & 7,03 & 0,02 & $\mathrm{~b}$ \\
\hline $\mathrm{T} 2$ & 7,14 & 0,02 & $\mathrm{c}$ \\
\hline $\mathrm{T} 3$ & 7,28 & 0,01 & $\mathrm{~d}$
\end{tabular}

Nota: (a) $\mathrm{N}=60$, Resultado de $\mathrm{pH}$ en cuatro tratamientos de biol a partir de restos de trucha y estiércol vacuno: control (C), tratamiento 1 (T1), tratamiento 2 (T2), tratamiento 3 (T3). Medias y error estándar (SE) son mostradas. Datos analizados con Anova Tukey entre tratamientos (letras en minúscula).

Tabla 4. Producción de biogás total

\begin{tabular}{cccc}
\hline & \multicolumn{3}{c}{ Biogás total (L) } \\
\cline { 2 - 4 } Tratamientos $^{\mathbf{a}}$ & Media & SE & Tukey \\
\cline { 2 - 4 } & 72,63 & 1,57 & $\mathrm{a}$ \\
\hline $\mathrm{C}$ & 95,57 & 1,63 & $\mathrm{~b}$ \\
\hline $\mathrm{T} 1$ & 121,57 & 2,03 & $\mathrm{C}$ \\
\hline $\mathrm{T} 2$ & 159,30 & 2,52 & $\mathrm{~d}$ \\
\hline
\end{tabular}

Nota: (a) $\mathrm{N}=3$. Biogás total (L) en cuatro tratamientos de biol a partir de restos de trucha y estiércol vacuno: control (C), tratamiento 1 (T1), tratamiento 2 (T2) y tratamiento 3 (T3). Medias y error estándar (SE) son mostradas. Datos analizados con Anova Tukey entre tratamientos (letras en minúscula).
Para el caso del nitrógeno se obtuvo que el tratamiento T3 fue el de mayor cantidad de nitrógeno $(6600 \mathrm{mg} / \mathrm{l}$ ). Se obtuvieron diferencias significativas: $F(3,8)=7,47$, $p=0,010$. La prueba de Tukey demostró que hay una diferencia significativa de la concentración de nitrógeno en el tratamiento 3 al compararlo con el control. El tratamiento 3 también tuvo una diferencia significativa al ser comparado con el tratamiento 1. Sin embargo,

Tabla 5. Prueba de Tukey para las diferentes concentraciones de nutrientes de los tratamientos evaluados

\begin{tabular}{|c|c|c|c|c|}
\hline Concentraciones & Tratamientos ${ }^{\mathrm{a}}$ & Media & SE & Tukey \\
\hline \multirow{4}{*}{$\begin{array}{l}\text { Concentración de } \\
\text { nitrógeno }\left(\mathrm{mg}^{\left.-\mathrm{l}^{-1}\right)}\right.\end{array}$} & C & 5206,45 & 200,18 & $\mathrm{a}$ \\
\hline & $\mathrm{T} 1$ & 5414,89 & 208,17 & $\mathrm{a}$ \\
\hline & $\mathrm{T} 2$ & 5769,44 & 49,01 & $a b$ \\
\hline & T3 & 6600,00 & 340,45 & $b$ \\
\hline \multirow{4}{*}{$\begin{array}{l}\text { Concentración de } \\
\text { potasio }\left(\mathrm{mg} . \mathrm{l}^{-1}\right)\end{array}$} & C & 961,67 & 56,74 & a \\
\hline & $\mathrm{T} 1$ & 1463,33 & 84,13 & $a b$ \\
\hline & $\mathrm{T} 2$ & 1563,33 & 89,18 & $\mathrm{~b}$ \\
\hline & T3 & 1136,67 & 182,79 & $a b$ \\
\hline \multirow{4}{*}{$\begin{array}{l}\text { Concentración de } \\
\left.\text { calcio (mg. } .^{-1}\right)\end{array}$} & $\mathrm{C}$ & 124,67 & 7,07 & a \\
\hline & $\mathrm{T} 1$ & 314,17 & 54,92 & $b$ \\
\hline & $\mathrm{T} 2$ & 1016,33 & 18,41 & $\mathrm{C}$ \\
\hline & T3 & 1603,33 & 27,28 & d \\
\hline \multirow{4}{*}{$\begin{array}{l}\text { Concentración de } \\
\text { magnesio }\left(\mathrm{mg}^{\left.-\mathrm{l}^{-1}\right)}\right.\end{array}$} & C & 49,50 & 1,26 & $a$ \\
\hline & $\mathrm{T} 1$ & 79,67 & 5,55 & a \\
\hline & $\mathrm{T} 2$ & 303,33 & 18,56 & b \\
\hline & $\mathrm{T} 3$ & 563,33 & 39,30 & $\mathrm{C}$ \\
\hline \multirow{4}{*}{$\begin{array}{l}\text { Concentración de } \\
\text { sodio }\left(\mathrm{mg}^{\left.-l^{-1}\right)}\right.\end{array}$} & $C$ & 358,83 & 26,33 & a \\
\hline & $\mathrm{T} 1$ & 573,33 & 8,82 & $b$ \\
\hline & $\mathrm{T} 2$ & 775,00 & 12,58 & c \\
\hline & T3 & 1408,33 & 60,85 & d \\
\hline \multirow{4}{*}{$\begin{array}{l}\text { Concentración de } \\
\text { ácidos húmicos (\%) }\end{array}$} & $\mathrm{C}$ & 0,00 & 0,00 & $a$ \\
\hline & $\mathrm{T} 1$ & 0,05 & 0,05 & a \\
\hline & $\mathrm{T} 2$ & 0,61 & 0,04 & $b$ \\
\hline & T3 & 1,06 & 0,10 & c \\
\hline \multirow{4}{*}{$\begin{array}{l}\text { Concentración de } \\
\text { ácidos fúlvicos (\%) }\end{array}$} & $\mathrm{C}$ & 0,60 & 0,07 & $\mathrm{a}$ \\
\hline & $\mathrm{T} 1$ & 0,47 & 0,07 & $a$ \\
\hline & $\mathrm{T} 2$ & 0,46 & 0,04 & $\mathrm{a}$ \\
\hline & $\mathrm{T} 3$ & 1,39 & 0,32 & $b$ \\
\hline \multirow{4}{*}{$\begin{array}{l}\text { Concentración de } \\
\text { huminas (\%) }\end{array}$} & $\mathrm{C}$ & 0,07 & 0,02 & $a$ \\
\hline & $\mathrm{T} 1$ & 0,05 & 0,02 & $a$ \\
\hline & $\mathrm{T} 2$ & 0,57 & 0,07 & b \\
\hline & $\mathrm{T} 3$ & 0,48 & 0,10 & b \\
\hline
\end{tabular}

Nota: (a) $\mathrm{N}=3$. Concentración de nitrógeno, potasio, calcio, magnesio, sodios (mg.l-1), ácidos húmicos, ácidos fúlvicos y huminas (\%) en cuatro tratamientos de biol a partir de restos de trucha y estiércol vacuno: control (C), tratamiento 1 (T1), tratamiento 2 (T2), tratamiento 3 (T3). Medias y error estándar (SE) son mostrados. Datos analizados con Anova Tukey entre tratamientos (letras en minúscula). 
el tratamiento 2 resultó no significativo entre el tratamiento 1, el tratamiento 3 y el control.

El potasio presentó diferencias significativas entre tratamientos: $F(3,8)=6,085, p=0,018$. La prueba de Tukey demostró que la concentración de potasio fue significativa en el tratamiento 2 al compararlo con el control. Sin embargo, el tratamiento 1 y el tratamiento 3 resultaron no significativos entre el tratamiento 2 y el control.

El calcio presentó diferencias significativas determinadas por Anova de un factor: $F(3,8)=443,173$, $p<0,001$. La prueba de Tukey demostró que el tratamiento 3 , el tratamiento 2 y el tratamiento 1 fueron significativos al compararlos con los demás tratamientos y el control (ver tabla 5).

Se ha encontrado diferencia significativa entre tratamientos para la concentración de magnesio. Esta diferencia significativa fue determinada por Anova de un factor: $F(3,8)=23,78, p<0,001$. La prueba de Tukey demostró que hay una diferencia significativa de la concentración de magnesio en el tratamiento 3 y el tratamiento 2 al compararlos con todos los tratamientos y el control. Sin embargo, el tratamiento 1 resultó no significativo entre el control.

Lo mismo sucede para la concentración del sodio, que muestra una diferencia significativa entre tratamientos determinada por Anova de un factor: $F(3,8)=177,008$, $p<0,001$. La prueba de Tukey demostró que la concentración de sodio entre el tratamiento 3, el tratamiento 2 y el tratamiento 1 fue significativa en comparación con los demás tratamientos y control.

Los ácidos húmicos, fúlvicos y huminas evaluados en la investigación fueron analizados estadísticamente mediante un análisis de varianza entre tratamientos y arrojaron diferencias significativas $(p<0,05)$ entre tratamientos para la concentración de ácidos húmicos determinada por Anova de un factor: $F(3,8)=23,78$, $p<0,001$. La prueba de Tukey demostró que hay una diferencia significativa de la concentración de ácidos húmicos en el tratamiento 3 y el tratamiento 2 al compararlos con todos los tratamientos y el control. Sin embargo, el tratamiento 1 no presentó diferencias significativas entre el control.

De igual forma, la concentración de ácidos fúlvicos tiene diferencias significativas entre tratamientos determinadas por Anova de un factor: $F(3,8)=7,140$, $p=0,012$. La prueba de Tukey demostró que hay una diferencia significativa de la concentración de ácidos fúlvicos en el tratamiento 1, el tratamiento 2 y el control al compararlos con el tratamiento 3. Por otro lado, el tratamiento 1 , el tratamiento 2 y el control no presentaron diferencias significativas.

Finalmente, existe una diferencia significativa entre tratamientos para la concentración de huminas determinada por Anova de un factor: $F(3,8)=17,614$, $p=0,001$. La prueba de Tukey demostró que hay una diferencia significativa de la concentración de huminas en el tratamiento 3 al compararlo con el tratamiento 1 y el control. Lo mismo sucede para el tratamiento 2 al compararlo con el tratamiento 1 y el control. Sin embargo, el tratamiento 1 y el control resultaron no significativos al compararse entre sí. Lo mismo sucedió para el tratamiento 2 y el tratamiento 3 al realizar las comparaciones entre sí.

\section{Relación entre producción de biogás y composición del biol}

La correlación de Pearson se realizó para determinar si existe una relación entre la producción de biogás y macronutrientes primarios. Se obtuvo una correlación fuerte y positiva con una significancia $(r=0,78$, $\mathrm{N}=12, \mathrm{p}=0,03$ ) entre la concentración de nitrógeno y la producción de biogás; una correlación muy débil positiva y no significativa ( $r=0,136, N=12, p=0,674)$ entre la concentración de potasio y la producción de biogás; una correlación muy fuerte y positiva con una significancia ( $r$ $=0,981, \mathrm{~N}=12, \mathrm{p}<0,01$ ) entre la concentración de calcio y la producción de biogás; una muy fuerte y positiva con una significancia $(r=0,96, N=12, p<0,01)$ entre la concentración de magnesio y la producción de biogás; finalmente, una correlación muy fuerte y positiva con una significancia $(r=0,977, N=12, p<0,01)$ entre la concentración de sodio y la producción de biogás.

Para el caso de los ácidos húmicos, fúlvicos y huminas se tuvo una correlación muy fuerte y positiva con una significancia ( $r=0,946, N=12, p<0,01)$ entre la concentración de ácidos húmicos y la producción de biogás; una fuerte y positiva con una significancia ( $r=0,678, N=12, p=0,015)$ entre la concentración deácidos fúlvicos y la producción de biogás; finalmente, una correlación fuerte y positiva con una significancia ( $r=0,753$, $\mathrm{N}=12, \mathrm{p}=0,005$ ) entre la concentración de huminas y la producción de biogás, como se observa en la figura 3.

\section{Discusión}

\section{Variación de pH y temperatura semanal}

El pH para todos los tratamientos tiende a la neutralidad en un rango de 6,94 a 7,29, lo que, según la Organización de las Naciones Unidas para la Alimentación y la Agricultura (FAO, por sus siglas en inglés) (2011), favorece el proceso de degradación anaeróbica.

De acuerdo con Cárdenas (2009), se tiene una mayor producción de ácido orgánico en los digestores por causa de la actividad de las bacterias acidogénicas y de los acetoclásticos metanógenos que convierten los ácidos orgánicos en metano y COV (Venkata Mohan, 2008). Ello hace que el $\mathrm{pH}$ disminuya y que, a medida que el proceso de fermentación continúa, la concentración de amoniaco aumente gradualmente por la acción de las bacterias catabolizadoras de proteínas, neutralice el ácido orgánico y mantenga el valor de $\mathrm{pH}$ en la neutralidad. 

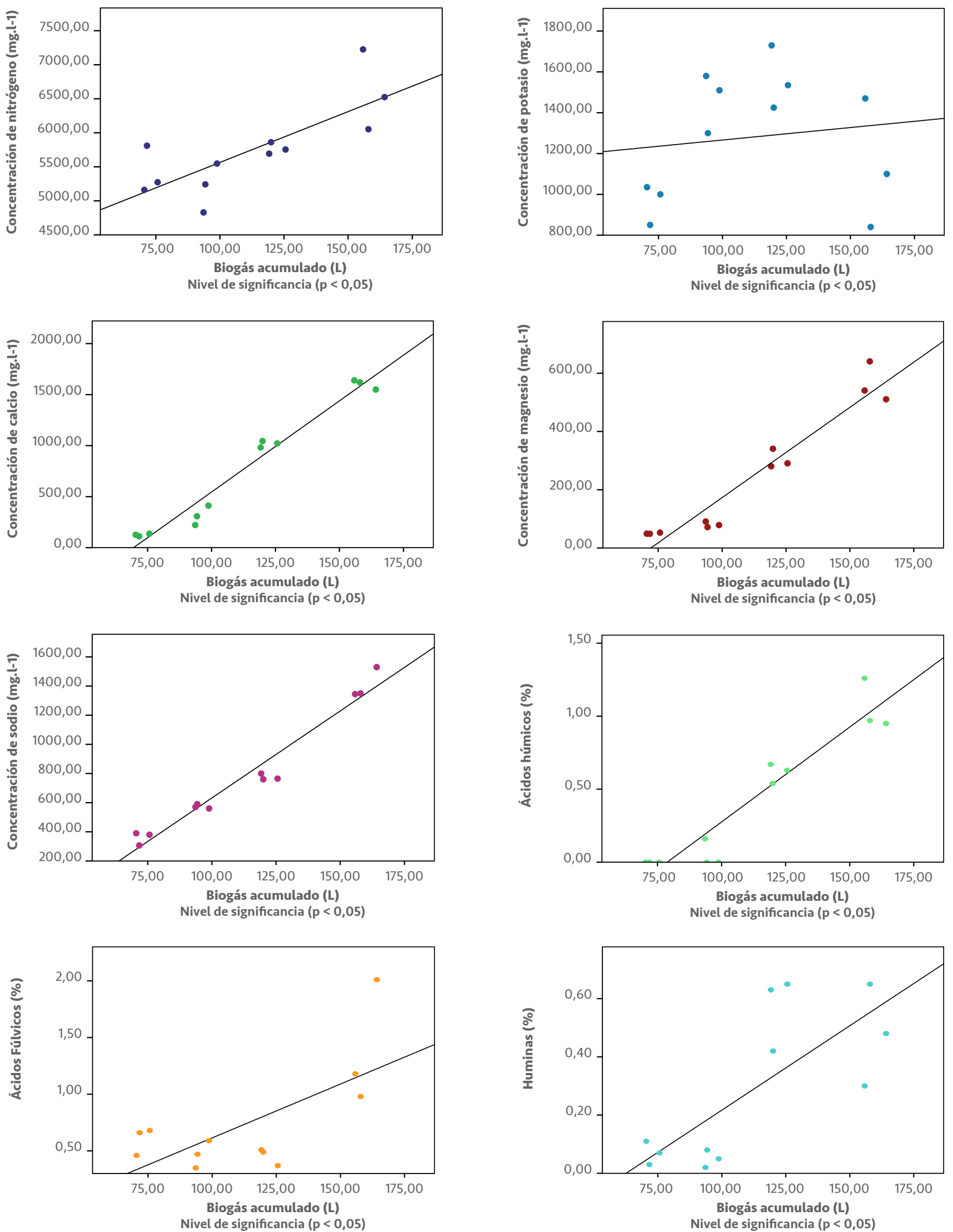

Figura 3. Correlación de Pearson entre concentración de nitrógeno, potasio, calcio, magnesio, sodio (mg.l-1), ácidos húmicos, ácidos fúlvicos, huminas (\%) y producción de biogás (L) 
Por otro lado, la temperatura de todos los tratamientos fue de régimen mesofílico, con un rango de temperatura ambiental de $26 \pm 4{ }^{\circ} \mathrm{C}$ correspondiente a temporada de verano (febrero-abril). Según la FAO (2011), las temperaturas elevadas implican una reducción en los tiempos de retención y un aumento en la solubilidad de la mayoría de las sales, de manera que la materia orgánica es más accesible para los microorganismos y esto motiva el incremento en la velocidad de la digestión del volumen del material en los tanques.

\section{Variación de producción de biogás entre tratamientos}

Según Eiroa et al. (2012), los restos de trucha contienen altos contenidos de ácidos grasos de cadena larga, lo que ocasiona una acumulación del contenido de lípidos, así como una posible inhibición de los tratamientos por un exceso de producción de amoniaco formado por las altas concentraciones de nitrógeno que tienen los restos de peces. Además, reducen hasta en un 50 \% la producción de metano. El tratamiento control de la presente investigación obtuvo los resultados más bajos con respecto a la producción de biogás debido a este factor limitante que tienen los restos de peces para una adecuada digestión anaerobia, a la menor carga microbiana inicial y a la accesibilidad de los nutrientes por tener un porcentaje de humedad más elevado.

Se puede mencionar que el volumen de biogás obtenido a partir de los restos de trucha y estiércol de vaca del T3 es mayor que el obtenido por el control al finalizar la investigación. Esto se debe a que la adición de estiércol vacuno en diferentes cantidades mejoró la relación carbono-nitrógeno de los tratamientos y las elevadas temperaturas permitieron un aumento en la velocidad de reacción (FAO, 2011).

\section{Variación de composición de biol entre tratamientos}

Los resultados obtenidos con respecto a la concentración de nitrógeno son bastante altos en comparación con otros autores que usaron diferentes materias orgánicas a descomponer, como Cárdenas (2009), que tuvo valores de $676,67 \mathrm{mg} . \mathrm{l}^{-1}$ y $814,33 \mathrm{mg} . \mathrm{l}^{-1}$ al usar estiércol de vaca y mezcla de restos orgánicos con estiércol de vaca, respectivamente, lo que demostró que el estiércol de vaca favorece en el aumento de concentración de nitrógeno. Sin embargo, los resultados obtenidos son casi tres veces más elevados a los comparados con la literatura, debido a que en un estudio realizado por Gostincar (1998) los restos de peces tienen un alto contenido de nitrógeno, porque son ricos en proteínas. Esto se comprueba con los resultados del tratamiento que tenía vísceras de truchay otros componentes en el estudio de Jiménez (2012), donde la concentración de nitrógeno fue de 2950,19 mg. $\mathrm{l}^{-1}$, similar a la obtenida en la presente investigación.

Para el caso de la concentración de potasio, calcio, magnesio y sodio se pudo observar que su concentración aumenta conforme se agrega estiércol de vaca. Esto se complementa con lo propuesto por Quipuzco, Baldeón y Tang (2011), que al usar dos mezclas de estiércol de vaca la más alta concentración de estos nutrientes fue obtenida por el tratamiento que contenía mayor cantidad de estiércol, a diferencia de la mezcla con menor cantidad de estiércol. Ello demuestra que existe una relación sinérgica positiva al adicionar estiércol de vacuno a los restos de trucha.

Asimismo, la concentración de nitrógeno, potasio, calcio y magnesio de los bioles en los tratamientos obtenidos de la presente investigación fueron mayores a las concentraciones propuestas por Álvarez (2010), que obtuvo 920 mg.l-1, 860,4 mg. $\mathrm{l}^{-1}, 112,1 \mathrm{mg} . \mathrm{l}^{-1}$ y 54,7 mg. $\mathrm{l}^{-1}$, respectivamente. Se demostró así que los restos de trucha tienen un buen contenido de macronutrientes para que puedan ser aplicados como abonos orgánicos.

Finalmente, la concentración de ácidos húmicos, fúlvicos y huminas de los tratamientos evaluados también tuvieron una relación sinérgica positiva al agregar mayores cantidades de estiércol de vaca. Esto se debe a que existe una mayor concentración de estos elementos en el estiércol, materia en descomposición, aguas de ríos y lagunas (Shimabukuro, 1996).

\section{Relación entre la producción de biogás y la composición del biol}

Durante el periodo de 10 semanas se tuvieron tres tratamientos con diferentes cantidades de estiércol y restos de trucha, además de un control que evaluó la cantidad de biogás obtenido y la concentración de macronutrientes al término del periodo, cuando la producción de biogás casi había finalizado. En función de esto, los diferentes tratamientos obtuvieron distintas concentraciones de macronutrientes y producción acumulada de biogás, cuyo orden para ambas variables fue $\mathrm{T} 3>\mathrm{T} 2>\mathrm{T} 1>$ Control.

Existe una correlación fuerte positiva entre la producción de biogás en litros y las concentraciones de nutrientes como nitrógeno, calcio, magnesio, sodio, ácidos húmicos, ácidos fúlvicos y huminas. Esta magnitud de correlación se basó en el estudio realizado por Evans (1996), que menciona que el coeficiente de correlación tiene un signo y una magnitud que determina si es una correlación fuerte positiva o negativa. De esta forma se puede afirmar que, de acuerdo con Eiroa et al. (2012), la codigesión anaerobia, al usar restos de trucha y estiércol vacuno, tendrá una mayor producción de biogás y una mayor concentración de nutrientes en los bioles.

\section{Conclusiones}

La adición de estiércol de vaca a los restos de trucha en diferentes cantidades mejoró la relación carbononitrógeno y el proceso de degradación anaeróbica de los biodigestores. Se obtuvo así un mejor control del $\mathrm{pH}$ desde 
el momento de arranque (rangos entre 6,94 y 7,29). Por otro lado, la producción de biogás aumenta en función de la adición de estiércol de vaca. Este sustrato es el más adecuado para una codigestión anaerobia positiva de los restos de trucha, por lo que el T3 obtuvo los más altos volúmenes de biogás acumulados (159,3 litros).

Finalmente, existe una correlación positiva fuerte entre la producción de biogás y biol obtenidos en este proyecto. Los restos de trucha tienen un alto potencial de aprovechamiento de las actividades acuícolas como abono orgánico, ya que mejoran de manera sinérgica la fermentación anaeróbica, la composición del abono orgánico y la producción de biogás al adicionar estiércol vacuno.

\section{Fuente de financiamiento}

Agradecimientos a la empresa Peruvian Andean Trout S. A. C., que colaboró enormemente en la financiación del desarrollo de la fase experimental de esta investigación, demostrando la excelente calidad humana de sus miembros y su compromiso para promover la investigación y el cuidado del medioambiente.

\section{Contribución de autoría}

OG se encargó de la ejecución experimental del proyecto, así como de la estadística, las discusiones y las conclusiones presentadas. LG se encargó de la asesoría técnica para el desarrollo de este proyecto. Ambos autores aprobaron la versión final del texto.

\section{Potenciales conflictos de interés}

Los autores declaran que no existe ningún conflicto de interés.

\section{Referencias bibliográficas}

Álvarez, F. (2010). Preparación y uso del biol. Disponible en: http://www. semilla-austral.coop/wp-content/uploads/2018/06/65817533Preparacion-y-uso-del-biol.pdf

Cárdenas, G. (2009). Aislamiento térmico de biodigestor en planta de tratamiento de aguas servidas osorno. Tesis de Ingeniería Mecánica. Universidad Austral de Chile. Disponible en: http://cybertesis.uach. cl/tesis/uach/2009/bmfcic266a/doc/bmfcic266a.pdf

Castillo, G. (2012). Evaluación de codornaza y gallinaza de granjas avícolas para la producción de biogás y bioabono mediante digestión anaeróbica. Tesis de Ingeniería Ambiental. Universidad Nacional Agraria La Molina.

Centro de Estudios para el Desarrollo y la Participación. (2009). Manual de crianza de trucha (Oncorhynchus mykiss). Ragash: Antamina.

Comité de Operaciones del Sistema Interconectado Nacional. (2015). Resumen estadístico anual 2014. Lima: Subdirección de Gestión de la Información.

Contreras, E. S. (1994). Bioquímica de pescados e derivados. Jaboticabal: Fundação de Apoio a Pesquisa, Ensino e Extensão (Funep).
Eiroa, M., Costa, J. C., Alves, M. M., Kennes, C., Veiga, M. C. (2012). «Evaluation of biomethane potential of solid fish waste». Waste Management, 32, pp. 1347-1352. DOI: https://doi.org/10.1016/j. wasman.2012.03.020

Evans, J. D. (1996). Straightforward statistics for the behavioral sciences. Pacific Grove: Brooks/Cole Publishing.

Food and Agriculture Organization of the United Nations. (2011). Manual de biogás. Disponible en: http://www.fao.org/3/as400s/ as400s.pdf

Gostincar, J. (1998). Técnicas agrícolas en cultivos extensivos. Madrid: Idea Books.

Jiménez Mideros, J. (2012). Elaboración de abono orgánico líquido fermentado (biol), a partir de vísceras de trucha arco iris (Oncorhynchus mykiis), de los criaderos piscícolas de la parroquia de Tufiño. Tulcán: Universidad Politécnica Estatal del Carchi. Disponible en: http:// repositorio.upec.edu.ec/handle/123456789/15

Ministerio de Agricultura y Riego. (2011). Biodigestores en el Perú. Guía de principales experiencias desarrolladas en el país. Disponible en: http://agroaldia.minagri.gob.pe/biblioteca/download/pdf/ manuales-boletines/bioenergia/biodigestores.pdf

Ministerio del Ambiente. (2013). VI informe anual de residuos sólidos municipales y no municipales. Lima: Ministerio del Ambiente.

Palomino, V. (2007). Tratamiento de residuos sólidos domésticos mediante biodigestores para la obtención de biogás y biol. Lima: Universidad Nacional Agraria La Molina.

Plan Nacional de Desarrollo Acuícola. (2010). Plan Nacional de Desarrollo Acuícola 2010-2021. Lima: Ministerio de la Producción.

Quipuzco, L., Baldeón, W. y Tang, O. (2011). «Evaluación de la calidad de biogás y biol a partir de dos mezclas de estiércol de vaca en biodigestores tubulares de PVC». Revista del Instituto de Investigaciones de la Facultad de Geología, Minas, Metalurgia y Ciencias Geográficas, 27(14), pp. 1-8. Disponible en: https:// revistasinvestigacion.unmsm.edu.pe/index.php/iigeo/article/ view/690

Restrepo Rivera, J. (2001). Elaboración de abonos orgánicos, fermentados y biofertilizantes foliares. San José: Instituto Interamericano de Cooperación para la Agricultura.

Servicio Nacional de Sanidad Agraria. (2015). Estadísticas de producción orgánica a nivel nacional 2015. Disponible en: https:// www.senasa.gob.pe/senasa/descargasarchivos/2014/12/Situaciónde-la-Producción-Orgánica-Nacional-2015.pdf

Shimabukuro, M. (1996). Efecto de la aplicación de ácidos húmicos y fertilizantes foliares en el rendimiento y calidad de vainita. Tesis de Ingeniería Agrícola. Universidad Nacional Agraria La Molina.

Soria, M., Ferrera-Cerrato, R., Barra, J., González, G., Santos, J., Gómez, L., et al. (2001). «Producción de biofertilizantes mediante biodigestión de excreta líquida de cerdo». Terra, 19(4), pp. 353-362. Disponible en: https://www.redalyc.org/pdf/573/57319408.pdf

Venkata Mohan, S. (2008). «Fermentative hydrogen production with simultaneous wastewater treatment: Influence of pretreatment and system operating conditions». Journal of Scientific \& Industrial Research, 67, pp. 950-961. Disponible en: https://pdfs.semanticscholar. org/1ea0/45078d7321fb7c330da217da56738e208792.pdf 